\title{
An association study in the Taiwan Biobank elicits the GABAA receptor genes GABRB3, GABRA5, and GABRG3 as candidate loci for sleep duration in the Taiwanese population
}

Sheue-Jane Hou, ${ }^{1,2}$, Shih-Jen Tsai, ${ }^{3,4}$, Po-Hsiu Kuo ${ }^{5,6}$, Wan-Yu Lin ${ }^{5}$, Yu-Li Liư , Albert C. Yang ${ }^{8,9}$, Eugene $\operatorname{Lin}^{10,11,12^{*}}$ and Tsuo-Hung Lan ${ }^{1,13^{*}}$

\begin{abstract}
Background: Gamma-aminobutyric acid type A (GABAA) receptors mainly mediate the effects of gamma-aminobutyric acid, which is the primary inhibitory neurotransmitter in the central nervous system. Abundant evidence suggests that GABAA receptors play a key role in sleep-regulating processes. No genetic association study has explored the relationships between GABAA receptor genes and sleep duration, sleep quality, and sleep timing in humans.

Methods: We determined the association between single-nucleotide polymorphisms (SNPS) in the GABAA receptor genes GABRA1, GABRA2, GABRB3, GABRA5, and GABRG3 and sleep duration, sleep quality, and sleep timing in the Taiwan Biobank with a sample of 10,127 Taiwanese subjects. There were 10,142 subjects in the original study cohort. We excluded 15 subjects with a medication history of sedative-hypnotics.

Results: Our data revealed an association of the GABRB3-GABRA5-GABRG3 gene cluster with sleep duration, which has not been previously identified: rs79333046 (beta $\left.=-0.07 ; P=1.21 \times 10^{-3}\right)$ in $G A B R B 3$, rs 189790076 (beta $=0.92$; $\left.P=1.04 \times 10^{-3}\right)$ in GABRA5, and rs 147619342 (beta $\left.=-0.72 ; P=3.97 \times 10^{-3}\right)$ in GABRG3. The association between rs 189790076 in GABRA5 and sleep duration remained significant after Bonferroni correction. A variant (rs12438141) in GABRB3 was also found to act as a potential expression quantitative trait locus. Additionally, we discovered interactions between variants in the GABRB3-GABRA5-GABRG3 gene cluster and lifestyle factors, such as tea and coffee consumption, smoking, and physical activity, that influenced sleep duration, although some interactions became nonsignificant after Bonferroni correction. We also found interactions among GABRB3, GABRA5, and GABRG3 that affected sleep duration. Furthermore, we identified an association of rs7165524 (beta $=-0.06 ; P=2.20 \times 10^{-3}$ ) in GABRA5 with sleep quality and an association of rs 79465949 (beta $=-0.12 ; P=3.95 \times 10^{-3}$ ) in GABRB3 with sleep timing, although these associations became nonsignificant after Bonferroni correction. However, we detected no evidence of an association of individual SNPs in GABRA1 and GABRA2.
\end{abstract}

\footnotetext{
*Correspondence: lines@uw.edu; tosafish@gmail.com

${ }^{10}$ Department of Biostatistics, University of Washington, 3980 15th

Avenue NE, Box 351617, Seattle, WA 98195, USA

${ }^{13}$ Tsaotun Psychiatric Center, Ministry of Health and Welfare, Nantou,

Taiwan

Full list of author information is available at the end of the article
}

(C) The Author(s) 2021. Open Access This article is licensed under a Creative Commons Attribution 4.0 International License, which permits use, sharing, adaptation, distribution and reproduction in any medium or format, as long as you give appropriate credit to the original author(s) and the source, provide a link to the Creative Commons licence, and indicate if changes were made. The images or other third party material in this article are included in the article's Creative Commons licence, unless indicated otherwise in a credit line to the material. If material is not included in the article's Creative Commons licence and your intended use is not permitted by statutory regulation or exceeds the permitted use, you will need to obtain permission directly from the copyright holder. To view a copy of this licence, visit http://creativecommons.org/licenses/by/4.0/. The Creative Commons Public Domain Dedication waiver (http://creativeco mmons.org/publicdomain/zero/1.0/) applies to the data made available in this article, unless otherwise stated in a credit line to the data. 
Conclusions: Our results indicate that rs 189790076 in GABRA5 and gene-gene interactions among GABRB3, GABRA5, and GABRG3 may contribute to sleep duration in the Taiwanese population.

Keywords: Biobank, GABAA receptor gene, Diverse populations, GABRB3-GABRA5-GABRG3 gene cluster, Gene-gene interaction, Gene-environment interaction, Sleep duration

\section{Background}

Principally, gamma-aminobutyric acid type A (GABAA) receptors are recognized as a crucial factor in sleepregulating processes in the central nervous system [1]. In drug design and discovery to date, many of the drugs prescribed for insomnia (i.e., sleeping pills or sedativehypnotic drugs) exert effects on GABAA receptors in terms of the physiology and pharmacology of sleep [2-5]. Molecular biology studies have demonstrated two modes of neuronal transmission in the brain that are distributed evenly, namely inhibition mediated by gamma-aminobutyric acid (GABA) and excitation mediated by glutamate $[6,7]$. Moreover, the effects of GABA, the principal inhibitory neurotransmitter, are primarily mediated by GABAA receptors in the central nervous system [8]. Increasing evidence suggests that GABAA receptors play a pivotal role in regulating sleep and wake cyclical patterns (i.e., circadian rhythms) in humans $[9,10]$. Numerous studies have implied a link between sleep disorders (i.e., circadian rhythm misalignment; for example, short/ too little $[\leq 6 \mathrm{~h}$ per day] or long/too much sleep [ $>9 \mathrm{~h}$ per day] [11]) and alterations in the function of GABAA receptors $[9,12,13]$. Sleep is crucial for improving quality of life, and sleep duration is characterized as the total amount of sleep during a 24-h daily physiological cycle in the biological system [14, 15]. Few genetic variants have been revealed to be associated with sleep duration despite heritability accounting for approximately $40 \%$ of sleep duration $[16,17]$.

GABAA receptors are coded by GABAA receptor genes such as gamma-aminobutyric acid type A receptor subunit alpha1 (GABRA1). Studies have suggested a connection between GABAA receptor genes and sleeprelated behaviors [18-21]. For example, Tsai et al. [19] indicated a significant association between zolpideminduced complex sleep behaviors and the $\mathrm{A} 15 \mathrm{G}$ variant in the GABAA receptor gene GABRA1 in the Taiwanese population. Choi et al. [18] also reported that the rs4263535 single-nucleotide polymorphism (SNP) in the GABAA receptor gene GABRA1 contributes to sleep induction time during sedation induction using intravenous midazolam in the Korean population. In addition, Blednov et al. [21] suggested that Gabra1 or Gabra2 knockout mice (i.e., with the deletion or loss of the Gabra1 or Gabra2 gene) exhibited significantly reduced sleep time, which was induced by GABAA agonists. Moreover, a study implied that Gabra1 knockout mice spent markedly less time in rapid eye movement sleep but markedly more time in non-rapid eye movement sleep [20].

The GABRB3-GABRA5-GABRG3 gene cluster consists of the GABAA receptor genes GABRB3, GABRA5, and GABRG3 located on chromosome 15q12, which encode subunits beta3, alpha5, and gamma3, respectively [22, 23]. The GABRB3-GABRA5-GABRG3 gene cluster has been the focus of attention for many years, and studies have suggested that it is involved in various psychiatric and neurological diseases with comorbid sleep disorders, such as panic disorder, autism spectrum disorders, Angelman and Prader-Willi syndrome, and epilepsy [24-28].

Although several other genes are associated with sleepregulation processes [29], to the best of our knowledge, no study has addressed the impact of GABAA receptor genes such as GABRA1, GABRA2, GABRB3, GABRA5, and $G A B R G 3$ on sleep duration in humans. GABAA receptor genes have been speculated to be linked to sleep duration because the encoded products of these genes (i.e., GABAA receptors) play a central role in the sleep and wake timing of the circadian rhythm system in humans $[2,4,7]$. A study also reported that lifestyle factors such as physical activity are linked to health outcomes [30]. In addition, several studies [31-35] in the Taiwan Biobank have conducted interaction analyses on various traits, including gene-gene, gene-environment, gene-age, and gene-gender interactions. One study suggested that interactions between the RORA gene and lifestyle factors, including tea consumption, coffee consumption, physical activity, alcohol consumption, and smoking, may influence sleep duration [31]. Based on the aforementioned considerations, we conducted the first genetic association study on the relationships between GABAA receptor genes such as GABRA1, GABRA2, GABRB3, GABRA5, and GABRG3 and sleep duration, sleep quality, and sleep timing in Taiwanese subjects from the Taiwan Biobank. Although there are other GABAA receptor genes, this pilot study focused on these five genes (i.e., GABRA1, GABRA2, GABRB3, GABRA5, and GABRG3) due to more studies on these genes based on previous literature [18-28]. Moreover, we performed interaction analyses on sleep duration as per previously described methods [31-35]. 


\section{Materials and methods}

\section{Subjects}

Taiwanese participants were recruited from the Taiwan Biobank for the present study [32-40]. The Taiwan Biobank contains specimens and their related data from the general Taiwanese population from multiple recruitment centers across Taiwan from 2012 to the present (ongoing) [32-40]. Focused on providing collaboration opportunities for researchers, the Taiwan Biobank is mainly funded by the Taiwanese government, and it facilitates public health-related research projects related to local common chronic diseases [36].

Participants were included if they met the following criteria: (1) able to perform activities of daily living and (2) self-reported Taiwanese ancestry [37]. Individuals with a history of cancer or nonresidents of Taiwan were excluded [37]. Our original study cohort comprised 10,142 Taiwanese subjects. We further excluded individuals with a medication history of sedative-hypnotics $(\mathrm{n}=15)$. In total, there were 10,127 individuals in our final study cohort.

Self-reported data on "sleep duration", "sleep quality" and "sleep timing" from the Taiwan Biobank questionnaire were used in this study [31]. Sleep duration was defined as the average number of hours slept in a 24-h period [31]. Classifications of " 1 " for very poor, " 2 " for poor, " 3 " for fair, " 4 " for good and " 5 " for very good were used as measures for average sleep quality [31]. For sleep timing, six subgroups were defined based on the individual's usual sleep onset time: (1) 0-4 AM, (2) 4-8 AM, (3) 8-12 AM, (4) 12-16 PM, (5) 16-20 PM, and (6) 20-24 PM, which is a standard method in the Taiwan Biobank as previously described [31]. As part of the questionnaire, participants were also asked if they were current tea/alcohol/coffee drinkers, if they were current smokers, and if they had performed any recent physical activity [31]. Participants who were currently drinking coffee at least three times per week were classified as current coffee drinkers [31]. Similarly, participants who were currently drinking tea at least one time per day were classified as current tea drinkers [31]. Participants with an alcohol intake of over $150 \mathrm{~mL}$ per week for more than 6 months were classified as current alcohol drinkers [31]. Participants who had been smoking for more than 6 months were classified as current smokers [31]. Performing physical activity was defined as having exercised more than three times per week, for over $30 \mathrm{~min}$ each time [31]. Status of chronic disorders was defined as whether the participant had had the following chronic disorders: arthritis, asthma, diabetes, and/or heart disease.

The Institutional Review Board of the Taiwan Biobank granted ethical approval for the study before it was conducted (approval number: 201506095RINC). Each subject signed an approved informed consent form. Relevant regulations and guidelines were applied to all experiments performed.

\section{Genotyping}

With the manufacturer's instructions, DNA was isolated from blood samples via QIAmp DNA blood kit (Qiagen, Valencia, CA, USA). Agarose gel electrophoresis determined the quality of isolated genomic DNA, with evaluation of the quantity made using spectrophotometry [41]. We used the custom Taiwan Biobank chips for SNP genotyping conducted on the Axiom GenomeWide Arrray Plate System (Affymetrix, Santa Clara, CA, USA). SNPs on the Axiom Genome-Wide CHB 1 Array (Affymetrix) and the Human Exome BeadChip (Illumina, Inc., San Diego, CA, USA) were used to create custom Taiwan Biobank chips in order to efficiently extract maximal genetic information from Taiwanese samples [37]. Both the custom Taiwan Biobank chip and the Axiom Genome-Wide CHB 1 Array were used to genotype 70 unrelated Taiwanese individuals in order to accurately examine the performance of the chip previously mentioned. A high average concordance rate of $99.55 \%$ was achieved as a result for the SNPs in the 70 individuals [37].

In the present study, we searched for GABAA receptor-associated variants by referring to the complete list of GABAA receptor genes (such as GABRA1, GABRA2, $G A B R B 3, G A B R A 5$, and GABRG3) available in the custom Taiwan Biobank chips. Furthermore, the following quality control criteria were adopted for excluding SNPs from subsequent analysis [32, 42]: failure to achieve Hardy-Weinberg equilibrium (with a $P$ value less than $0.05)$, minor allele frequency $(\mathrm{MAF})<1 \%$, or a genotyping call rate less than 95\%. After exclusion according to those criteria, we established independent SNPs that are not in linkage disequilibrium to each other using the LDlink tool [43] with an Asian reference panel. Finally, we obtained two SNP panels. The first SNP panel covered 43 SNPs from the following three GABAA receptor genes: $G A B R B 3, G A B R A 5$, and GABRG3. The second SNP panel covered five SNPs from the following two GABAA receptor genes: GABRA1 and GABRA2.

\section{Statistical analysis}

Chi-squared test was used to evaluate categorical data. Differences in the means of two continuous variables were compared by conducting the Student's $t$ test. Furthermore, we investigated the association of the investigated SNP with sleep duration, sleep quality, and sleep timing using linear regression analysis, adjusting for age, gender, physical activity, coffee consumption, tea consumption, smoking, alcohol consumption, body 
mass index (BMI), and status of chronic disorders as previously described [34, 44]. Sleep quality and sleep timing, captured as categorical variables, were analyzed as continuous variables in the genetic association analysis. We performed dominant, recessive, and genotypic genetic association tests using PLINK [42]. The dominant model was calculated using the major allele homozygous genotypes as references (i.e., major allele homozygous carriers vs. heterozygous and minor allele homozygous carriers grouped together). The recessive model was calculated using the heterozygous and major allele homozygous genotypes as references (i.e., heterozygous and major allele homozygous carriers grouped together vs. minor allele homozygous carriers). A chisquared goodness-of-fit test with 1 degree of freedom (i.e. the number of genotypes minus the number of alleles) was used to assess the genotype frequencies for Hardy-Weinberg equilibrium [31]. Multiple testing was adjusted with Bonferroni correction [31]. For example, we divided 0.05 by the number of independent SNPs (i.e., 43) in the first SNP panel to obtain the significant $P$ value $\left(P<0.05 / 43=1.16 \times 10^{-3}\right) . \quad$ Mean \pm standard deviation were shown as a presentation of data [31].

In addition, we used the most significant SNP (in the dominant, recessive, or genotypic model) in a specific GABAA receptor gene of the GABRB3-GABRA5GABRG3 gene cluster (such as GABRB3, GABRA5, and GABRG3) to perform interaction analyses using $\mathrm{R}$, which was a similar approach to that used in a recent study [31]. To investigate gene-gene and gene-environment interactions, we used multiple linear regression models and provided covariates such as age, gender, physical activity, coffee consumption, tea consumption, smoking, alcohol consumption, BMI, and status of chronic disorders in our interaction analyses based on previous literature [31]. The individuals were also stratified into five age groups by decade of life $(30 \mathrm{~s}, 40 \mathrm{~s}, 50 \mathrm{~s}, 60 \mathrm{~s}$, and $70 \mathrm{~s})$, as previously described [31]. To investigate gene-age (using age groups) and gene-gender interactions, we used multiple linear regression models and provided covariates such as gender/age, physical activity, coffee consumption, tea consumption, smoking, alcohol consumption, BMI, and status of chronic disorders in our interaction analyses as previously described [31]. Moreover, we performed a stratified analysis for significant interactions. We also implemented interaction plots [45] to clarify whether interactions were significant in gene-environment interactions.

HaploReg (http://compbio.mit.edu/HaploReg) [46] was used to examine potential functional mechanisms, which means to estimate an SNP as an expression quantitative trait locus (eQTL).

\section{Results}

Taiwan Biobank sample

Table 1 presents the demographic characteristics of the study cohort, which comprised 10,127 individuals. The mean and the standard deviation values of sleep duration for overall, male, and female subjects were $6.6 \pm 1.1$, $6.7 \pm 1.1$, and $6.4 \pm 1.2 \mathrm{~h}$, respectively. In addition, the mean and standard deviation values of age for overall, male, and female subjects were $56.5 \pm 9.4,58.0 \pm 9.8$, and $56.1 \pm 9.2$ years, respectively.

\section{Novel variants for sleep duration in the GABRB3-GABRA5-GABRG3 gene cluster identified in the Taiwanese population}

Among the 43 SNPs assessed in this study, there were six SNPs in the GABRB3 gene, three SNPs in the GABRA5 gene, and four SNPs in the GABRG3 gene, which provided evidence of an association $(P<0.05)$ with sleep duration (Table 2).

As described in Table 2, we identified the most significant SNP in GABRB3, GABRA5, and GABRG3 as follows: the rs189790076 SNP (beta=0.92; $P=1.04 \times 10^{-3}$ ) in the intron region of the GABRA5 gene, the rs79333046 SNP (beta $=-0.07 ; \quad P=1.21 \times 10^{-3}$ ) in the intron region of the GABRB3 gene, and the rs147619342 SNP (beta $=-0.72 ; P=3.97 \times 10^{-3}$ ) in the intron region of

Table 1 Demographic characteristics of the study subjects

\begin{tabular}{llll}
\hline Characteristic & Overall & Male & Female \\
\hline No. of subjects, $n$ & 10,127 & 2,327 & 7,800 \\
Mean age \pm SD, years & $56.5 \pm 9.4$ & $58.0 \pm 9.8$ & $56.1 \pm 9.2$ \\
Sleep duration \pm SD, & $6.6 \pm 1.1$ & $6.7 \pm 1.1$ & $6.4 \pm 1.2$ \\
hours per day & & & \\
Sleep quality, n (\%) & & & \\
"1"for very poor & $1: 271(2.7)$ & $1: 37(1.6)$ & $1: 234(3.0)$ \\
"2"for poor & $2: 1,927(19.0)$ & $2: 355(15.3)$ & $2: 1,572(20.2)$ \\
"3"for fair & $3: 4,374(43.2)$ & $3: 950(40.8)$ & $3: 3,424(43.9)$ \\
"4"for good & $4: 2,140(21.1)$ & $4: 571(24.5)$ & $4: 1,569(20.1)$ \\
"5" for very good & $5: 1,412(13.8)$ & $5: 414(17.8)$ & $5: 998(12.8)$ \\
Sleep timing, $n(\%)$ & & & \\
(1) 0-4 AM & $(1): 2,071(20.5)$ & $(1): 440(18.9)$ & $(1): 1,631(20.9)$ \\
(2) 4-8 AM & $(2): 23(0.2)$ & $(2): 11(0.5)$ & $(2): 12(0.2)$ \\
(3) 8-12 AM & $(3): 20(0.2)$ & $(3): 11(0.5)$ & $(3): 9(0.1)$ \\
(4) 12-16 PM & $(4): 7(0.1)$ & $(4): 0(0.0)$ & $(4): 7(0.1)$ \\
(5) 16-20 PM & $(5): 27(0.3)$ & $(5): 9(0.4)$ & $(5): 18(0.2)$ \\
(6) 20-24 PM & $(6): 7,941(78.4)$ & $(6): 1,830(78.6)$ & $(6): 6,111(78.3)$
\end{tabular}

Data are presented as mean \pm standard deviation

Sleep quality is classified as " 1 " for very poor, " 2 " for poor, " 3 " for fair, " 4 " for good, and " 5 " for very good to evaluate the quality of usual sleep

Sleep timing is divided into six subgroups: (1) 0-4 AM, (2) 4-8 AM, (3) 8-12 AM, (4) 12-16 PM, (5) 16-20 PM, and (6) 20-24 PM based on usual sleep onset time $S D$ standard deviation 
Table 2 Linear regression models of associations between sleep duration and the GABAA receptor genes GABRB3, GABRA5, and GABRG3

\begin{tabular}{|c|c|c|c|c|c|c|c|c|c|c|c|c|c|c|c|}
\hline \multirow[t]{2}{*}{ Gene } & \multirow[t]{2}{*}{ Chr } & \multirow[t]{2}{*}{ SNP } & \multirow[t]{2}{*}{ A1 } & \multirow[t]{2}{*}{ A2 } & \multirow[t]{2}{*}{ Region } & \multirow[t]{2}{*}{ MAF } & \multicolumn{3}{|c|}{ Dominant model } & \multicolumn{3}{|c|}{ Recessive model } & \multicolumn{3}{|c|}{ Genotypic model } \\
\hline & & & & & & & Beta & SE & $P$ & Beta & SE & $P$ & Beta & SE & $P$ \\
\hline \multirow[t]{14}{*}{ GABRB3 } & \multirow[t]{14}{*}{15} & rs150101078 & A & G & Intron & 0.040 & 0.13 & 0.04 & $2.61 \times 10^{-3}$ & 0.01 & 0.28 & 0.985 & 0.01 & 0.14 & 0.956 \\
\hline & & rs12438141 & T & $C$ & Missense & 0.059 & 0.07 & 0.04 & 0.034 & -0.02 & 0.19 & 0.927 & 0.00 & 0.10 & 0.962 \\
\hline & & rs79565260 & A & G & Intron & 0.178 & 0.03 & 0.02 & 0.230 & 0.06 & 0.06 & 0.365 & 0.03 & 0.03 & 0.310 \\
\hline & & rs149646336 & T & C & Intron & 0.027 & 0.00 & 0.05 & 0.939 & 0.15 & 0.43 & 0.728 & 0.07 & 0.21 & 0.728 \\
\hline & & rs74591460 & $C$ & A & Intron & 0.099 & -0.01 & 0.03 & 0.861 & 0.22 & 0.11 & 0.040 & 0.11 & 0.05 & 0.043 \\
\hline & & rs79465949 & T & $C$ & Intron & 0.018 & 0.01 & 0.06 & 0.839 & 0.65 & 0.56 & 0.249 & 0.32 & 0.28 & 0.249 \\
\hline & & rs74004636 & T & G & Intron & 0.057 & -0.01 & 0.04 & 0.850 & -0.44 & 0.20 & 0.024 & -0.22 & 0.10 & 0.024 \\
\hline & & rs79333046 & A & G & Intron & 0.213 & -0.07 & 0.02 & $1.21 \times 10^{-3}$ & -0.07 & 0.06 & 0.207 & -0.05 & 0.03 & 0.088 \\
\hline & & rs76635931 & G & T & Intron & 0.014 & 0.09 & 0.07 & 0.187 & -1.08 & 1.13 & 0.335 & NA & NA & NA \\
\hline & & rs111863451 & G & $\mathrm{T}$ & Intron & 0.027 & -0.02 & 0.05 & 0.685 & 0.80 & 0.40 & 0.045 & 0.40 & 0.20 & 0.046 \\
\hline & & rs145333058 & $\mathrm{T}$ & $C$ & Intron & 0.015 & 0.02 & 0.07 & 0.716 & -0.54 & 0.80 & 0.495 & -0.27 & 0.40 & 0.495 \\
\hline & & rs146937293 & $C$ & T & Intron & 0.043 & 0.01 & 0.04 & 0.770 & 0.09 & 0.23 & 0.709 & 0.04 & 0.12 & 0.707 \\
\hline & & rs77328500 & G & A & Intron & 0.077 & -0.02 & 0.03 & 0.442 & 0.03 & 0.15 & 0.860 & 0.01 & 0.08 & 0.879 \\
\hline & & rs74653915 & $C$ & $\mathrm{~T}$ & Intron & 0.016 & -0.11 & 0.06 & 0.103 & 0.02 & 0.80 & 0.980 & 0.01 & 0.40 & 0.984 \\
\hline \multirow[t]{8}{*}{ GABRA5 } & \multirow[t]{8}{*}{15} & rs78575803 & A & G & Intron & 0.101 & 0.02 & 0.03 & 0.498 & 0.11 & 0.10 & 0.297 & 0.06 & 0.05 & 0.287 \\
\hline & & rs7165524 & T & C & Intron & 0.270 & -0.02 & 0.02 & 0.311 & -0.02 & 0.04 & 0.721 & -0.01 & 0.02 & 0.573 \\
\hline & & rs 80063633 & A & C & Intron & 0.151 & 0.00 & 0.02 & 0.949 & 0.15 & 0.07 & 0.045 & 0.07 & 0.04 & 0.049 \\
\hline & & rs140148185 & A & G & Intron & 0.090 & -0.01 & 0.03 & 0.809 & 0.39 & 0.13 & $2.73 \times 10^{-3}$ & 0.19 & 0.06 & $3.05 \times 10^{-3}$ \\
\hline & & rs146013014 & G & A & Intron & 0.026 & 0.07 & 0.05 & 0.182 & 0.55 & 0.40 & 0.163 & 0.28 & 0.20 & 0.161 \\
\hline & & rs189790076 & $C$ & G & Intron & 0.016 & 0.06 & 0.06 & 0.316 & 1.84 & 0.56 & $1.05 \times 10^{-3}$ & 0.92 & 0.28 & $1.04 \times 10^{-3}$ \\
\hline & & rs140319999 & T & $C$ & Intron & 0.018 & 0.10 & 0.06 & 0.103 & -0.17 & 0.80 & 0.827 & -0.09 & 0.40 & 0.831 \\
\hline & & rs34560927 & $\mathrm{T}$ & C & Intron & 0.372 & 0.00 & 0.02 & 0.988 & 0.06 & 0.03 & 0.091 & 0.02 & 0.02 & 0.174 \\
\hline \multirow[t]{21}{*}{ GABRG3 } & \multirow[t]{21}{*}{15} & rs34984550 & G & $C$ & Intron & 0.024 & 0.00 & 0.05 & 0.941 & -0.18 & 0.56 & 0.752 & -0.09 & 0.28 & 0.752 \\
\hline & & rs 140670 & A & $\mathrm{T}$ & Intron & 0.293 & 0.01 & 0.02 & 0.664 & -0.03 & 0.04 & 0.455 & -0.01 & 0.02 & 0.586 \\
\hline & & rs147619342 & T & C & Intron & 0.017 & -0.08 & 0.06 & 0.208 & -1.45 & 0.50 & $4.02 \times 10^{-3}$ & -0.72 & 0.25 & $3.97 \times 10^{-3}$ \\
\hline & & rs12903002 & $\mathrm{T}$ & $C$ & Intron & 0.088 & 0.02 & 0.03 & 0.490 & 0.01 & 0.13 & 0.921 & 0.01 & 0.07 & 0.901 \\
\hline & & rs1017364 & G & A & Intron & 0.266 & 0.03 & 0.02 & 0.217 & -0.01 & 0.04 & 0.813 & 0.00 & 0.02 & 0.946 \\
\hline & & rs150722146 & A & G & Intron & 0.026 & -0.05 & 0.05 & 0.282 & -0.29 & 0.56 & 0.604 & -0.15 & 0.28 & 0.601 \\
\hline & & rs138047237 & A & G & Intron & 0.013 & 0.06 & 0.07 & 0.384 & 1.30 & 0.80 & 0.103 & 0.65 & 0.40 & 0.103 \\
\hline & & rs734254 & A & G & Intron & 0.034 & -0.11 & 0.05 & 0.018 & -0.33 & 0.34 & 0.325 & -0.17 & 0.17 & 0.316 \\
\hline & & rs12593313 & G & $C$ & Intron & 0.022 & 0.00 & 0.06 & 0.989 & -0.01 & 0.43 & 0.990 & 0.00 & 0.21 & 0.990 \\
\hline & & rs140853604 & T & C & Intron & 0.031 & -0.01 & 0.05 & 0.789 & 0.32 & 0.37 & 0.387 & 0.16 & 0.19 & 0.389 \\
\hline & & rs7173587 & T & $C$ & Intron & 0.157 & -0.06 & 0.02 & 0.023 & 0.08 & 0.07 & 0.288 & 0.03 & 0.04 & 0.419 \\
\hline & & rs143682098 & G & A & Intron & 0.053 & -0.02 & 0.04 & 0.679 & -0.12 & 0.25 & 0.645 & -0.06 & 0.13 & 0.641 \\
\hline & & rs17739682 & G & A & Intron & 0.072 & 0.02 & 0.03 & 0.620 & 0.12 & 0.17 & 0.489 & 0.06 & 0.08 & 0.483 \\
\hline & & rs144369966 & $C$ & T & Intron & 0.018 & 0.06 & 0.06 & 0.316 & -0.29 & 0.65 & 0.653 & -0.14 & 0.32 & 0.656 \\
\hline & & rs67338000 & A & G & Intron & 0.449 & -0.04 & 0.02 & 0.068 & 0.00 & 0.03 & 0.859 & -0.01 & 0.02 & 0.401 \\
\hline & & rs8028000 & C & T & Intron & 0.014 & -0.14 & 0.07 & 0.041 & -1.64 & 0.80 & 0.039 & -0.82 & 0.40 & 0.039 \\
\hline & & rs61507711 & T & $C$ & Intron & 0.097 & 0.02 & 0.03 & 0.582 & 0.10 & 0.12 & 0.384 & 0.05 & 0.06 & 0.376 \\
\hline & & rs9302372 & A & G & Intron & 0.042 & 0.02 & 0.04 & 0.670 & -0.08 & 0.23 & 0.740 & -0.04 & 0.12 & 0.745 \\
\hline & & rs4555109 & T & G & Intron & 0.222 & 0.04 & 0.02 & 0.104 & 0.01 & 0.05 & 0.825 & 0.01 & 0.03 & 0.624 \\
\hline & & rs149403614 & A & G & Intron & 0.035 & 0.00 & 0.04 & 0.994 & 0.05 & 0.40 & 0.894 & 0.03 & 0.20 & 0.894 \\
\hline & & rs28399529 & G & A & $3^{\prime}$ UTR & 0.022 & 0.00 & 0.06 & 0.965 & -0.25 & 0.65 & 0.705 & -0.12 & 0.33 & 0.705 \\
\hline
\end{tabular}

Analysis was obtained after adjustment for covariates, including age, gender, physical activity, coffee consumption, tea consumption, smoking, alcohol consumption, body mass index (BMI), and status of chronic disorders

$P$ values $<0.05$ represent the significant values and are shown in bold

A1 minor allele, A2 major allele, GABAA gamma-aminobutyric acid type A, Beta Beta coefficients, Chr chromosome, MAF minor allele frequency, NA not available, SE standard error 
the GABRG3 gene. Moreover, rs189790076 in GABRA5 remained significant after applying Bonferroni correction $\left(P<0.05 / 43=1.16 \times 10^{-3}\right)$.

Furthermore, rs140148185 (beta $=0.39 ; P=2.73 \times 10^{-3}$; intron region) in $G A B R A 5$ and rs 150101078 (beta $=0.13$; $P=2.61 \times 10^{-3}$; intron region) in GABRB3 exhibited a borderline significant trend with sleep duration (Table 2). In addition, we identified a nominal association of sleep duration with rs80063633 in GABRA5, four SNPs (rs12438141, rs74591460, rs74004636, and rs111863451) in GABRB3, and three SNPs (rs734254, rs7173587, and rs8028000) in GABRG3 (Table 2).

In addition, the rs $12438141 \mathrm{SNP}$ in the missense region of the GABRB3 gene was shown to be a potential eQTL in various tissues such as nerve and adipose tissues; this was reported previously [47].

\section{Gene-environment interactions in the GABRB3-GABRA5-GABRG3 gene cluster for sleep duration identified in the Taiwanese population}

As described in Table 3, we identified significant interaction models between GABRB3 rs79333046 and lifestyle factors, including physical activity $\left(P=2.08 \times 10^{-3}\right)$, coffee consumption $\left(P=3.26 \times 10^{-4}\right)$, tea consumption $\left(P=3.66 \times 10^{-3}\right)$, and smoking $(P=0.0345)$. The effects of these models concerning physical activity and coffee consumption remained significant after Bonferroni correction $\left(P<0.05 / 15=3.33 \times 10^{-3}\right)$. These analysis results indicated probable gene-environment interactions

Table 3 Gene-environment interaction models identified by multiple linear regression analysis

\begin{tabular}{ll}
\hline Interaction model & P value \\
\hline GABRB3 rs79333046, physical activity & $2.08 \times 10^{-3}$ \\
GABRB3 rs79333046, coffee consumption & $3.26 \times 10^{-4}$ \\
GABRB3 rs79333046, tea consumption & $3.66 \times 10^{-3}$ \\
GABRB3 rs79333046, smoking & 0.0345 \\
GABRB3 rs79333046, alcohol consumption & $\mathrm{NS}$ \\
GABRA5 rs189790076, physical activity & $\mathrm{NS}$ \\
GABRA5 rs189790076, coffee consumption & $3.42 \times 10^{-3}$ \\
GABRA5 rs189790076, tea consumption & $\mathrm{NS}$ \\
GABRA5 rs189790076, smoking & $9.35 \times 10^{-3}$ \\
GABRA5 rs189790076, alcohol consumption & 0.0134 \\
GABRG3 rs147619342, physical activity & $\mathrm{NS}$ \\
GABRG3 rs147619342, coffee consumption & 0.0112 \\
GABRG3 rs147619342, tea consumption & $4.59 \times 10^{-3}$ \\
GABRG3 rs147619342, smoking & 0.0423 \\
GABRG3 rs147619342, alcohol consumption & $\mathrm{NS}$ \\
\hline An &
\end{tabular}

Analysis was obtained after adjustment for covariates, including age, gender, physical activity, coffee consumption, tea consumption, smoking, alcohol consumption, body mass index (BMI), and status of chronic disorders NS nonsignificant between $G A B R B 3$ and lifestyle factors such as physical activity and coffee consumption, respectively, which affected sleep duration. Additional file 2: Table S1 and Additional file 1: Figure S1 present the stratified analysis results and interaction plots for these two significant interactions, respectively. However, the plots and the stratified analysis did not show similar trends. While there was no variation of sleep duration for the genotypes of GABRB3 rs79333046 in terms of physical activity and coffee consumption in the interaction plots (Additional file 1: Figure S1 (A) and (B), respectively), there was no such a non-variable trend of the effect sizes in the stratified analysis for these two interactions (Additional file 2: Table S1).

In addition, we identified significant interaction models between GABRA5 rs189790076 and lifestyle factors, including coffee consumption $\left(P=3.42 \times 10^{-3}\right)$, smoking $\left(P=9.35 \times 10^{-3}\right)$, and alcohol consumption $(P=0.0134$; Table 3). However, the effects of these models concerning these lifestyle factors did not remain significant after Bonferroni correction $\left(P<0.05 / 15=3.33 \times 10^{-3}\right)$.

Moreover, we identified significant interaction models between GABRG3 rs147619342 and lifestyle factors, including coffee consumption $(P=0.0112)$, tea consumption $\left(P=4.59 \times 10^{-3}\right)$, and smoking $(P=0.0423$; Table 3). However, the effects of these models did not remain significant after Bonferroni correction $\left(P<0.05 / 15=3.33 \times 10^{-3}\right)$.

Gene-gene interactions in the GABRB3-GABRA5-GABRG3 gene cluster for sleep duration identified in the Taiwanese population

As described in Table 4, we identified significant interaction models involving GABRB3 rs79333046 and GABRA5 rs189790076 $\left(P=2.01 \times 10^{-3}\right), G A B R A 5$ rs 189790076 and GABRG3 rs147619342 $\left(P=1.05 \times 10^{-3}\right)$, and GABRB3 rs79333046 and GABRG3 rs147619342 $\left(P=4.68 \times 10^{-3}\right)$. The effects of these models concerning the GABRB3GABRA5-GABRG3 gene cluster remained significant after Bonferroni correction $(P<0.05 / 3=0.0167)$. These analysis results indicated probable gene-gene interactions among these three genes, which affected sleep

Table 4 Gene-gene interaction models identified by multiple linear regression analysis

\begin{tabular}{ll}
\hline Interaction model & P value \\
\hline GABRB3 rs79333046, GABRA5 rs189790076 & $2.01 \times 10^{-3}$ \\
GABRA5 rs189790076, GABRG3 rs147619342 & $1.05 \times 10^{-3}$ \\
GABRB3 rs79333046, GABRG3 rs147619342 & $4.68 \times 10^{-3}$
\end{tabular}

Analysis was obtained after adjustment for covariates, including age, gender, physical activity, coffee consumption, tea consumption, smoking, alcohol consumption, body mass index (BMI), and status of chronic disorders 
duration. Additional file 3: Table S2 presents the stratified analysis results for these significant interactions.

\section{Gene-gender and gene-age interactions in the GABRB3-GABRA5-GABRG3 gene cluster for sleep duration identified in the Taiwanese population}

We identified significant interaction models involving GABRB3 rs79333046 and gender $\left(P=8.14 \times 10^{-4}\right)$, GABRA5 rs189790076 and gender $\left(P=3.79 \times 10^{-4}\right)$, and GABRG3 rs147619342 and gender $\left(P=1.41 \times 10^{-3}\right)$. In addition, there were significant interaction models involving $G A B R B 3$ rs79333046 and age groups $\left(P=3.58 \times 10^{-10}\right)$ as well as GABRA5 rs189790076 and age groups $\left(P=2.24 \times 10^{-10}\right)$. Additional file 4: Table S3 presents the stratified analysis results for these significant interactions.

\section{Novel variants for sleep quality and sleep timing in the GABRB3-GABRA5-GABRG3 gene cluster identified in the Taiwanese population}

The association of the GABA receptor genes GABRB3, $G A B R A 5$, and GABRG3 on sleep quality and sleep timing was further investigated. Among the 43 SNPs investigated in the present study, we identified six SNPs in the GABRB3-GABRA5-GABRG3 gene cluster to be associated $(P<0.05)$ with sleep quality (Additional file 5: Table S4). The most significant SNP was the rs7165524 SNP (beta $=-0.06 ; P=2.20 \times 10^{-3}$ ) in the intron region of the GABRA5 gene.

In addition, we identified six SNPs in the GABRB3GABRA5-GABRG3 gene cluster to be associated $(P<0.05)$ with sleep timing (Additional file 6: Table S5). The most significant SNP was the rs79465949 SNP (beta $=-0.12 ; P=3.95 \times 10^{-3}$ ) in the intron region of the $G A B R B 3$ gene.

\section{No link of sleep duration with GABRA1 and GABRA2 in the Taiwanese population}

Finally, the association between sleep duration and GABA receptor genes such as GABRA1 and GABRA2 was explored. Among the five SNPs (including the two SNPs in GABRA1 and three SNPs in GABRA2) examined in the present study, no evidence of an association $(P<0.05)$ with sleep duration was identified (Additional file 7: Table S6).

The association of the GABA receptor genes GABRA1 and $G A B R A 2$ with sleep quality and sleep timing was further investigated. Among the five SNPs examined in the present study, we identified two (rs76707584 and rs16851626) in the GABRA2 gene to be associated $(P<0.05)$ with sleep quality (Additional file 8 : Table S7). In addition, no evidence of an association of the five
SNPs $(P<0.05)$ with sleep timing was identified (data not shown).

\section{Discussion}

The majority of genetic association studies for sleep duration have been conducted in European populations; it has been suggested that genetic association studies in globally underrepresented and diverse populations, such as the Taiwanese population, have great potential to identify novel biomarkers for common traits such as sleep duration across the human genome [48]. We conducted the first genetic association study of sleep duration, sleep quality, and sleep timing using the GABAA receptor genes GABRA1, GABRA2, GABRB3, GABRA5, and GABRG3 in the Taiwan Biobank.

In the present study, we found an association of sleep duration with SNPs in the GABRB3-GABRA5-GABRG3 gene cluster on chromosome 15 that has not been previously reported, such as rs79333046 in GABRB3, rs189790076 in GABRA5, and rs147619342 in GABRG3. To the best of our knowledge, this is the first study to report a probable association between sleep duration and the GABAA receptor genes GABRB3, GABRA5, and GABRG3. GABRB3, GABRA5, and GABRG3, which are all located on chromosome $15 \mathrm{q} 12$ and encode the alpha5, beta3, and gamma3 subunits of GABAA receptors, respectively $[49,50]$. Common in many neurological and psychiatric disorders in humans, GABAA receptors have been indicated to be involved in sleep disorders and sleep disturbances [2, 4, 7]. And these are encoded products of the three genes as a gene cluster namely the GABRB3-GABRA5-GABRG3 gene cluster, a promising candidate locus for sleep duration. Furthermore, it has been reported that the GABRB3-GABRA5-GABRG3 gene cluster may lead to increased susceptibility to a variety of neurological and psychiatric disorders with comorbid sleep disturbances, such as panic disorder, autism spectrum disorders, Angelman and Prader-Willi syndrome, and epilepsy [24-28]. For instance, Hodge et al. [25] reported that SNPs in the GABAA receptor genes $G A B R B 3$ and $G A B R A 5$ were associated with panic disorder in a Caucasian population. Notably, sleep-related behaviors such as sleep disturbance and sleep disorders are associated with patients with psychiatric diagnoses such as panic disorder [51].

We identified 13 SNPs in total in the GABRB3GABRA5-GABRG3 gene cluster to be associated with sleep duration (Table 2). We also found that the rs189790076 SNP in GABRA5 maintained a significant association with sleep duration after Bonferroni correction. The probable effects of these SNPs in the GABRB3GABRA5-GABRG3 gene cluster on sleep duration remain to be investigated. In the present study, we employed 
HaploReg to investigate potential functional mechanisms for these SNPs. Remarkably, we found that the rs12438141 SNP in the missense region of the GABRB3 gene served as a probable eQTL in nerve and adipose tissues [47] (see the Results section). A study reported that adipose tissue may play a key role in obstructive sleep apnea [52], a common sleep-related breathing disorder. Moreover, evidence of sensory nerve injury or dysfunction in obstructive sleep apnea was reported [53].

Based on the beta coefficient value for 79333046 in GABRB3 (Table 2; beta $=-0.07$, dominant model), the carriers of the AA and AG genotypes had lower sleep duration than the carriers of the GG genotype. Based on the beta coefficient value for rs189790076 in GABRA5 (Table 2; beta $=1.84$, recessive model), the carriers of the CC genotype had higher sleep duration than the carriers of the CG and GG genotypes. Based on the beta coefficient value for rs147619342 in GABRG3 (Table 2; beta = -1.45 , recessive model), the carriers of the TT genotype had lower sleep duration than the carriers of the TC and CC genotypes.

In the present study, our data revealed that geneenvironment interactions with the GABRB3-GABRA5GABRG3 gene cluster may be involved in the etiology of sleep duration. We found a significant association between the GABRB3-GABRA5-GABRG3 gene cluster and lifestyle factors, including tea consumption, coffee consumption, smoking, and physical activity. This interaction might be demonstrated through epigenetic modifications. However, some associations concerning lifestyle factors with GABRB3 rs79333046, GABRA5 rs189790076, and GABRG3 rs147619342 became nonsignificant after Bonferroni correction. There was also an inconsistent trend between the stratified analysis and the interaction plots for some significant associations concerning physical activity and coffee consumption with GABRB3 rs79333046. In addition to revealing the statistical significance of gene-environment interactions, we investigated the potential biological role underlying the interactions. The functional consequences of the interactions of the GABRB3-GABRA5-GABRG3 gene cluster with lifestyle factors in sleep duration remain to be elucidated. Similarly, Hou et al. [31] found a significant association between the circadian clock gene RORA and lifestyle factors, such as tea consumption, alcohol consumption, coffee consumption, smoking, and physical activity in affecting sleep duration in the Taiwanese population. A significant association between alcohol dependence and the GABAA receptor genes GABRB3 and GABRA5 was also revealed in a family-based association study in the Caucasian population [54]. Similarly, Dick et al. [55] observed that GABRG3 was associated with alcohol dependence in another family-based association study. In addition, Lopez et al. [56] reported that an interaction of caffeine with GABAA receptors may result in alterations in the transport function of GABAA receptors, and caffeine is the well-known stimulant in tea and coffee. Moreover, it has been suggested that major noncommunicable diseases are associated with the lack of physical activity worldwide [30]. However, to the best of our knowledge, no study has investigated the relationship between the GABRB3-GABRA5-GABRG3 gene cluster and lifestyle factors in affecting sleep duration. Based on previous findings [30, 31, 54-56], synergistic interactions between the GABRB3-GABRA5-GABRG3 gene cluster and lifestyle factors on sleep duration may be a hallmark of GABAA receptors in sleep and wake cyclical mechanisms.

We further inferred the epistatic effects of the GABRB3-GABRA5-GABRG3 gene cluster on sleep duration. To the best of our knowledge, our study is the first investigation of gene-gene interactions of the GABAA receptor genes GABRB3, GABRA5, and GABRG3 with sleep duration. Similarly, in a family-based association study, Ma et al. [57] identified potential gene-gene interactions of GABAA receptor genes GABRA4 and GABRB1 in the etiology of autism in a population with European ancestry. By contrast, Ashley-Koch et al. [58] detected no significant gene-gene interactions in the GABRB3-GABRA5-GABRG3 gene cluster that contributed to autism risk in a family-based association study in the Caucasian population. These findings suggest that the GABRA5 gene is closely related to the GABRB3 gene at the molecular level [22]. From a structural point of view, the GABRB3 gene is adjacent to the GABRA5 gene (approximately $100 \mathrm{~kb}$ apart) [59]. In a similar way, the GABRA5 gene is next to the GABRG3 gene (approximately $20 \mathrm{~kb}$ apart) [59]. In accordance with the aforementioned studies [22, 57-59], the present data indicate that potential allied reactions may exist among GABRB3, GABRA5, and GABRG3 in sleep duration, and the mechanisms may synergistically integrate with each other.

Furthermore, we found gene-gender and gene-age interactions of the GABRB3-GABRA5-GABRG3 gene cluster that influenced sleep duration. In accordance with our results, it has been indicated that sleep duration depends on both gender and age [31, 60]. Additionally, we found evidence of associations of the GABAA receptor genes GABRB3, GABRA5, and GABRG3 with sleep quality and sleep timing, although these associations were not statistically significant after Bonferroni correction. To the best of our knowledge, no other studies have explored the relationships of these genes with sleep quality and sleep timing. 
Finally, a link between sleep-related behaviors and the GABAA receptor genes GABRA1 and GABRA2 (or Gabra1 and Gabra2) has been found in various human and animal studies [18-21]. However, we did not detect an association of GABRA1 and GABRA2 with sleep duration in the Taiwanese population. In addition, we found evidence of associations of the GABAA receptor gene $G A B R A 2$ with sleep quality, although these associations were not statistically significant after Bonferroni correction.

This study has not only significant strengths but also some main weaknesses. A noteworthy strength is the considerable number of subjects from the Taiwan Biobank. By contrast, the major weakness is that the analysis was based on self-reported measures for sleep duration, timing, and quality. Furthermore, in our statistical analyses, adjustments were not made for an essential variable, namely shift work, due to a lack of such data.

\section{Conclusions}

In summary, our study suggests that rs189790076 in GABRA5 and gene-gene interactions among GABRB3, $G A B R A 5$, and $G A B R G 3$ may play a key role in sleep duration in the Taiwan Biobank. We also identified an association between rs7165524 in GABRA5 and sleep quality as well as an association between rs79465949 in GABRB3 and sleep timing, although these associations did not remain significant after Bonferroni correction. This genetic association study of sleep duration, sleep quality, and sleep timing in the Taiwan Biobank implicates the need for genetic association studies in worldwide underrepresented populations, such as the Taiwanese population. Even though substantial genetic association studies on common traits, such as sleep duration, have been conducted mainly in populations with European ancestry, such studies should be conducted in worldwide underrepresented populations for discovering novel loci. In the present study, we employed the Taiwan Biobank, which is the largest Taiwanese genetic database to date, to explore the SNPs related to sleep duration, sleep quality, and sleep timing in GABAA receptor genes. In future work, we expect to achieve more new genetic discoveries for common and/or complex traits in the Taiwanese population when the Taiwan Biobank proceeds to increase in volume.

\section{Abbreviations}

eQTL: Expression quantitative trait locus; GABA: Gamma-aminobutyric acid; GABAA: Gamma-aminobutyric acid type A; GABRA1: Gamma-aminobutyric acid type A receptor subunit alpha1; MAF: Minor allele frequency; SNPs:

Single-nucleotide polymorphisms.

\section{Supplementary Information}

The online version contains supplementary material available at https://doi. org/10.1186/s12920-021-01083-x.

Additional file 1 Figure S1. Interaction plots for significant interactions between GABRB3 rs79333046 and lifestyle factors.

Additional file $\mathbf{2}$ Table $\mathbf{S 1}$. Stratified analysis results for interactions between GABRB3 rs79333046 and lifestyle factors.

Additional file $\mathbf{3}$ Table S2. Stratified analysis results for gene-gene interactions.

Additional file 4 Table S3. Stratified analysis results for gene-gender and gene-age interactions.

Additional file $\mathbf{5}$ Table S4. Linear regression models of associations between sleep quality and six SNPs in GABAA receptor genes (e.g., GABRB3, GABRA5, and GABRG3) with evidence of an association ( $P<0.05)$.

Additional file 6 Table S5. Linear regression models of associations between sleep timing and six SNPs in GABAA receptor genes (e.g., GABRB3, GABRA5, and GABRG3) with evidence of an association $(P<0.05)$.

Additional file $\mathbf{7}$ Table S6. Linear regression models of associations between sleep duration and five SNPs in GABAA receptor genes (e.g., GABRA1 and GABRA2).

Additional file 8. Table S7. Linear regression models of associations between sleep quality and two SNPs in the GABAA receptor gene GABRA2 with evidence of an association $(P<0.05)$.

Acknowledgements

We thank Emily Ting and Wallace Academic Editing for English editing.

\section{Authors' contributions}

SJH contributed to the conception and drafting of the manuscript. SJT, PHK, WYL, YLL, and ACY contributed to data curation. EL performed data analysis and drafted the manuscript. THL contributed to the conception. All authors read and approved the final manuscript.

\section{Funding}

This work was supported by Taiwan's Ministry of Science and Technology (MOST 109-2634-F-075-001; SJT) and by Taipei Veterans General Hospital (V108D44-001-MY3-1; SJT).

\section{Availability of data and materials}

The data that support the findings of this study are available from the Taiwan Biobank. To apply for access to these third party data, please contact the Taiwan Biobank at biobank@gate.sinica.edu.tw.

\section{Declarations}

Ethical approval and consent to participate.

Ethical approval for the study was granted by the Institutional Review Board of the Taiwan Biobank before the study was conducted (approval number: 201506095RINC). Each subject signed the approved informed consent form. All experiments were performed in accordance with relevant guidelines and regulations.

\section{Consent for publication}

Not applicable.

Competing interests

The authors declare no conflicts of interest.

\section{Author details}

${ }^{1}$ Institute of Clinical Medicine, National Yang Ming Chiao Tung University, Taipei, Taiwan. ${ }^{2}$ Department of Psychiatry, Cheng Hsin General Hospital, Taipei, Taiwan. ${ }^{3}$ Department of Psychiatry, Taipei Veterans General Hospital, Taipei, Taiwan. ${ }^{4}$ Division of Psychiatry, National Yang Ming Chiao Tung University, Taipei, Taiwan. ${ }^{5}$ Department of Public Health, Institute of Epidemiology 
and Preventive Medicine, National Taiwan University, Taipei, Taiwan. ${ }^{6}$ Department of Psychiatry, National Taiwan University Hospital, Taipei, Taiwan. ${ }^{7}$ Center for Neuropsychiatric Research, National Health Research Institutes, Miaoli County, Taiwan. ${ }^{8}$ Division of Interdisciplinary Medicine and Biotechnology, Beth Israel Deaconess Medical Center, Harvard Medical School, Boston, MA

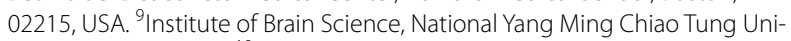
versity, Taipei, Taiwan. ${ }^{10}$ Department of Biostatistics, University of Washington, 3980 15th Avenue NE, Box 351617, Seattle, WA 98195, USA. ${ }^{11}$ Department of Electrical and Computer Engineering, University of Washington, Seattle, WA 98195, USA. ${ }^{12}$ Graduate Institute of Biomedical Sciences, China Medical University, Taichung, Taiwan. ${ }^{13}$ Tsaotun Psychiatric Center, Ministry of Health and Welfare, Nantou, Taiwan.

\section{Received: 10 March 2021 Accepted: 7 September 2021}

Published online: 16 September 2021

\section{References}

1. Nutt DJ, Stahl SM. Searching for perfect sleep: the continuing evolution of GABAA receptor modulators as hypnotics. J Psychopharmacol. 2010;24(11):1601-12.

2. Brickley SG, Franks NP, Wisden W. Modulation of GABAA receptor function and sleep. Curr Opin Physio. 2018;2:51-7.

3. Winsky-Sommerer R. Role of GABAA receptors in the physiology and pharmacology of sleep. Eur J Neurosci. 2009;29(9):1779-94.

4. Engin E, Benham RS, Rudolph U. An emerging circuit pharmacology of GABAA receptors. Trends Pharmacol Sci. 2018;39(8):710-32.

5. Sigel E, Ernst M. The benzodiazepine binding sites of GABAA receptors. Trends Pharmacol Sci. 2018;39(7):659-71.

6. Lancel M. Role of GABAA receptors in the regulation of sleep: initial sleep responses to peripherally administered modulators and agonists. Sleep. 1999;22(1):33-42.

7. Wisden W, Yu X, Franks N. GABA receptors and the pharmacology of sleep. Sleep Wake Neurobiol Pharmacol. 2019:279-304.

8. Johnston GA. GABAA receptor channel pharmacology. Curr Pharm Design. 2005;11(15):1867-85.

9. Brickley SG, Mody I. Extrasynaptic GABAA receptors: their function in the CNS and implications for disease. Neuron. 2012;73(1):23-34.

10. Steiger A. Neurochemical regulation of sleep. J Psychiatr Res. 2007;41(7):537-52.

11. Bin YS, Marshall NS, Glozier N. Sleeping at the limits: the changing prevalence of short and long sleep durations in 10 countries. Am J Epidemiol. 2013;177(8):826-33.

12. Möhler H. GABA A receptors in central nervous system disease: anxiety, epilepsy, and insomnia. J Recept Signal Trans. 2006;26(5-6):731-40.

13. Möhler H. GABA A receptor diversity and pharmacology. Cell Tissue Res. 2006;326(2):505-16.

14. Blair PS, Humphreys JS, Gringras P, Taheri S, Scott N, Emond A, et al. Childhood sleep duration and associated demographic characteristics in an English cohort. Sleep. 2012;35(3):353-60.

15. Krueger PM, Friedman EM. Sleep duration in the United States: a crosssectional population-based study. Am J Epidemiol. 2009;169(9):1052-63.

16. Barnes J, Meldrum RC. The impact of sleep duration on adolescent development: a genetically informed analysis of identical twin pairs. J Youth Adolesc. 2015;44(2):489-506.

17. de Castro JM. The influence of heredity on self-reported sleep patterns in free-living humans. Physiol Behavior. 2002;76(4-5):479-86.

18. Choi Y-J, Lee SY, Yang K-S, Park J-Y, Yoon SZ, Yoon SM. Polymorphism rs4263535 in GABRA1 intron 4 was related to deeper sedation by intravenous midazolam. J Int Med Res. 2015;43(5):686-98.

19. Tsai J-H, Yang P, Lin H-H, Cheng K-h, Yang Y-H, Wu M-T, et al. Association between GABAA receptor subunit gene cluster and zolpidem-induced complex sleep behaviors in Han Chinese. Sleep. 2013;36(2):197-202.

20. Ye G-L, Baker KB, Mason SM, Zhang W, Kirkpatrick L, Lanthorn TH, et al. GABA a receptor a1 subunit (Gabra1) knockout mice: review and new results. Transgenic and mutant tools to model brain disorders. Springer; 2010. pp. 65-90.

21. Blednov YA, Jung S, Alva H, Wallace D, Rosahl T, Whiting P-J, et al. Deletion of the alpha1 or beta2 Subunit of GABAA receptors reduces actions of alcohol and other drugs. J Pharmacol Exp Ther. 2003;304(1):30-6.
22. Greger $V$, Knoll J, Woolf E, Glatt K, Tyndale R, DeLorey T, et al. The $\gamma$-aminobutyric acid receptor $\gamma 3$ subunit gene (GABRG3) is tightly linked to the a5 subunit gene (GABRA5) on human chromosome 15q11-q13 and is transcribed in the same orientation. Genomics. 1995;26(2):258-64.

23. Culiat CT, Stubbs LJ, Montgomery CS, Russell LB, Rinchik EM. Phenotypic consequences of deletion of the gamma 3, alpha 5, or beta 3 subunit of the type A gamma-aminobutyric acid receptor in mice. Proc Natl Acad Sci. 1994;91(7):2815-8.

24. Kang J-Q, Barnes G. A common susceptibility factor of both autism and epilepsy: functional deficiency of GABA A receptors. J Autism Dev Disorders. 2013;43(1):68-79.

25. Hodges LM, Fyer AJ, Weissman MM, Logue MW, Haghighi F, Evgrafov O, et al. Evidence for linkage and association of GABRB3 and GABRA5 to panic disorder. Neuropsychopharmacology. 2014;39(10):2423-31.

26. McDougle CJ, Keary CJ. Sleep and EEG biomarkers as avenues toward new treatment approaches in Angelman syndrome. Neuropsychopharmacol Off Publ Am College Neuropsychopharmacol. 2020;45(1):238.

27. Frohlich J, Miller MT, Bird LM, Garces P, Purtell H, Hoener MC, et al. Electrophysiological phenotype in Angelman syndrome differs between genotypes. Biol Psychiatry. 2019;85(9):752-9.

28. Papandreou A, McTague A, Trump N, Ambegaonkar G, Ngoh A, Meyer E, et al. GABRB3 mutations: a new and emerging cause of early infantile epileptic encephalopathy. Dev Med Child Neurol. 2016;58(4):416-20.

29. Sehgal A, Mignot E. Genetics of sleep and sleep disorders. Cell. 2011;146(2):194-207.

30. Lee I-M, Shiroma EJ, Lobelo F, Puska P, Blair SN, Katzmarzyk PT, et al. Effect of physical inactivity on major non-communicable diseases worldwide: an analysis of burden of disease and life expectancy. The lancet. 2012;380(9838):219-29.

31. Hou S-J, Tsai S-J, Kuo P-H, Liu Y-L, Yang AC, Lin E, et al. An association study in the Taiwan Biobank reveals RORA as a novel locus for sleep duration in the Taiwanese population. Sleep Med. 2020;73:70-5.

32. Lin E, Kuo PH, Liu YL, Yang AC, Kao CF, Tsai SJ. Association and interaction of APOA5, BUD13, CETP, LIPA and health-related behavior with metabolic syndrome in a Taiwanese population. Sci Rep. 2016;6:36830.

33. Lin E, Kuo PH, Liu YL, Yang AC, Kao CF, Tsai SJ. Effects of circadian clock genes and environmental factors on cognitive aging in old adults in a Taiwanese population. Oncotarget. 2017;8(15):24088-98.

34. Lin E, Kuo PH, Liu YL, Yang AC, Kao CF, Tsai SJ. Effects of circadian clock genes and health-related behavior on metabolic syndrome in a Taiwanese population: evidence from association and interaction analysis. PLoS ONE. 2017;12(3):e0173861.

35. Lin E, Tsai SJ, Kuo PH, Liu YL, Yang AC, Kao CF. Association and interaction effects of Alzheimer's disease-associated genes and lifestyle on cognitive aging in older adults in a Taiwanese population. Oncotarget. 2017;8(15):24077-87.

36. Fan CT, Lin JC, Lee CH. Taiwan Biobank: a project aiming to aid Taiwan's transition into a biomedical island. Pharmacogenomics. 2008;9(2):235-46.

37. Chen $\mathrm{CH}$, Yang JH, Chiang CWK, Hsiung CN, Wu PE, Chang LC, et al. Population structure of Han Chinese in the modern Taiwanese population based on 10,000 participants in the Taiwan Biobank project. Hum Mol Genet. 2016;25(24):5321-31.

38. Lin E, Tsai SJ, Kuo PH, Liu YL, Yang AC, Kao CF, et al. The rs 1277306 variant of the REST gene confers susceptibility to cognitive aging in an elderly Taiwanese population. Dement Geriatr Cogn Disord. 2017;43(3-4):119-27.

39. Lin E, Tsai SJ, Kuo PH, Liu YL, Yang AC, Kao CF, et al. The ADAMTS9 gene is associated with cognitive aging in the elderly in a Taiwanese population. PLoS ONE. 2017;12(2):e0172440.

40. Lin E, Yang AC, Tsai SJ. Association between metabolic syndrome and cognitive function in old adults in a Taiwanese population. Taiwan J Psychiatry. 2017;31(3):232-40+iv.

41. Lin E, Kuo P-H, Liu Y-L, Yang AC, Tsai S-J. Polymorphisms of the DNA repair gene EXO1 modulate cognitive aging in old adults in a Taiwanese population. DNA Repair. 2019.

42. Purcell S, Neale B, Todd-Brown K, Thomas L, Ferreira MA, Bender D, et al. PLINK: a tool set for whole-genome association and population-based linkage analyses. Am J Hum Genet. 2007;81(3):559-75.

43. Machiela MJ, Chanock SJ. LDlink: a web-based application for exploring population-specific haplotype structure and linking correlated alleles of possible functional variants. Bioinformatics. 2015;31(21):3555-7. 
44. Lin E, Kuo P-H, Liu Y-L, Yang A, Tsai S-J. Association and interaction effects of interleukin-12 related genes and physical activity on cognitive aging in old adults in the Taiwanese population. Front Neurol. 2019;10:1065.

45. Wang $X$, Elston RC, Zhu X. The meaning of interaction. Hum Hered. 2010;70(4):269-77.

46. Ward LD, Kellis M. HaploReg v4: systematic mining of putative causal variants, cell types, regulators and target genes for human complex traits and disease. Nucleic Acids Res. 2016;44(D1):D877-81.

47. Consortium G. The Genotype-Tissue Expression (GTEx) pilot analysis: multitissue gene regulation in humans. Science. 2015;348(6235):648-60.

48. Wojcik GL, Graff M, Nishimura KK, Tao R, Haessler J, Gignoux CR, et al. Genetic analyses of diverse populations improves discovery for complex traits. Nature. 2019:1.

49. Glatt K, Glatt H, Lalande M. Structure and organization of GABRB3 and GABRA5. Genomics. 1997;41(1):63-9.

50. Kim Y, Glatt H, Xie W, Sinnett D, Lalande M. Human Y-aminobutyric acidtype A receptor a5 subunit gene (GABRA5): characterization and structural organization of the 5' flanking region. Genomics. 1997:42(3):378-87.

51. Malik S, Kanwar A, Sim LA, Prokop L, Wang Z, Benkhadra K, et al. The association between sleep disturbances and suicidal behaviors in patients with psychiatric diagnoses: a systematic review and meta-analysis. Syst Rev. 2014;3(1):18.

52. Ryan S, Arnaud C, Fitzpatrick SF, Gaucher J, Tamisier R, Pépin J-L. Adipose tissue as a key player in obstructive sleep apnoea. European Respiratory Review. 2019;28(152)

53. Boyd JH, Petrof BJ, Hamid Q, Fraser R, Kimoff RJ. Upper airway muscle inflammation and denervation changes in obstructive sleep apnea. Am J Respiratory Crit Care Med. 2004;170(5):541-6.

54. Song J, Koller DL, Foroud T, Carr K, Zhao J, Rice J, et al. Association of GABAA receptors and alcohol dependence and the effects of genetic imprinting. Am J Med Genet Part B Neuropsychiatr Genetics. 2003;117(1):39-45.

55. Dick DM, Edenberg HJ, Xuei X, Goate A, Kuperman S, Schuckit M, et al. Association of GABRG3 with alcohol dependence. Alcohol Clin Exp Res. 2004;28(1):4-9.

56. Lopez F, Miller LG, Greenblatt DJ, Kaplan GB, Shader RI. Interaction of caffeine with the GABAA receptor complex: alterations in receptor function but not ligand binding. Eur J Pharmacol Mol Pharmacol. 1989;172(6):453-9.

57. Ma D, Whitehead P, Menold M, Martin ER, Ashley-Koch A, Mei H, et al. Identification of significant association and gene-gene interaction of GABA receptor subunit genes in autism. Am J Hum Genet. 2005;77(3):377-88.

58. Ashley-Koch AE, Mei H, Jaworski J, Ma D, Ritchie MD, Menold M, et al. An analysis paradigm for investigating multi-locus effects in complex disease: examination of three GABAA receptor subunit genes on 15q11-q13 as risk factors for autistic disorder. Ann Hum Genet. 2006;70(3):281-92.

59. Sinnett D, Wagstaff J, Glatt K, Woolf E, Kirkness E, Lalande M. High-resolution mapping of the gamma-aminobutyric acid receptor subunit beta 3 and alpha 5 gene cluster on chromosome 15q11-q13, and localization of breakpoints in two Angelman syndrome patients. Am J Hum Genet. 1993;52(6):1216.

60. Allebrandt KV, Teder-Laving M, Akyol M, Pichler I, Müller-Myhsok B, Pramstaller $P$, et al. CLOCK gene variants associate with sleep duration in two independent populations. Biol Psychiatry. 2010;67(11):1040-7.

\section{Publisher's Note}

Springer Nature remains neutral with regard to jurisdictional claims in published maps and institutional affiliations.
Ready to submit your research? Choose BMC and benefit from:

- fast, convenient online submission

- thorough peer review by experienced researchers in your field

- rapid publication on acceptance

- support for research data, including large and complex data types

- gold Open Access which fosters wider collaboration and increased citations

- maximum visibility for your research: over $100 \mathrm{M}$ website views per year

At BMC, research is always in progress.

Learn more biomedcentral.com/submissions 